PROCEEDINGS OF THE

AMERICAN MATHEMATICAL SOCIETY

Volume 126, Number 9, September 1998, Pages 2557-2568

S $0002-9939(98) 04533-X$

\title{
ON UNIQUENESS OF $p$-ADIC MEROMORPHIC FUNCTIONS
}

\author{
ABDELBAKI BOUTABAA AND ALAIN ESCASSUT
}

(Communicated by William W. Adams)

\begin{abstract}
Let $K$ be a complete ultrametric algebraically closed field of characteristic zero, and let $\mathcal{M}(K)$ be the field of meromorphic functions in $K$. For all set $S$ in $K$ and for all $f \in \mathcal{M}(K)$ we denote by $E(f, S)$ the subset of $K \times \mathbb{N}^{*}$ : $\bigcup_{a \in S}\left\{(z, q) \in K \times \mathbb{N}^{*} \mid z\right.$ zero of order $q$ of $\left.f(z)-a\right\}$. After studying unique range sets for entire functions in $K$ in a previous article, here we consider a similar problem for meromorphic functions by showing, in particular, that, for every $n \geq 5$, there exist sets $S$ of $n$ elements in $K$ such that, if $f, g \in \mathcal{M}(K)$ have the same poles (counting multiplicities), and satisfy $E(f, S)=E(g, S)$, then $f=g$. We show how to construct such sets.
\end{abstract}

\section{INTRODUCTION AND THEOREMS}

Notation. $K$ will denote a complete ultrametric algebraically closed field of characteristic zero, and we denote by $\widehat{K}$ the one dimensional projective space over $K$ : $\widehat{K}=K \cup\{\infty\}$.

Given a field $L, L^{*}$ will denote $L \backslash\{0\}$.

We denote by $\mathcal{A}(K)$ the ring of entire functions in $K$ and by $\mathcal{M}(K)$ the field of meromorphic functions in all $K$.

For a subset $S$ of $K$ and $f \in \mathcal{M}(K)$ we denote by $E(f, S)$ the set in $K \times \mathbb{N}^{*}$ :

$$
\bigcup_{a \in S}\left\{(z, q) \in K \times \mathbb{N}^{*} \mid z \text { zero of order } q \text { of } f(z)-a\right\} .
$$

Besides, given a subset of $\widehat{K}$ containing $\{\infty\}$, we denote by $E(f, S)$ the subset of $K \times \mathbb{N}^{*}: E(f, S \cap K) \cup\{(z, q) \mid z$ pole of order $q$ of $f\}$.

Definition. Let $\mathcal{F}$ be a nonempty subset of $\mathcal{M}(K)$. A subset $S$ of $\widehat{K}$ is called a unique range set (a $U R S$ in short) for $\mathcal{F}$ if for any $f, g \in \mathcal{F}$ such that $E(f, S)=$ $E(g, S)$, one has $f=g$.

In the same way, a couple of sets $S, T$ in $\widehat{K}$ such that $S \cap T=\emptyset$ will be called $a b i$ $U R S$ for $\mathcal{F}$ if for any $f, g \in \mathcal{F}$ such that $E(f, S)=E(g, S)$ and $E(f, T)=E(g, T)$, one has $f=g$.

Remark 1. If a set $S$ is a URS for $\mathcal{A}(K)$ (resp. $\mathcal{M}(K)$ ), then for every nonconstant affine (resp. partial rational linear) function $h, h(S)$ also is a URS for $\mathcal{A}(K)$ (resp. for $\mathcal{M}(K)$ ). In the same way, if a couple of sets $(S, T)$ is a bi-URS for $\mathcal{A}(K)$ (resp.

Received by the editors October 22, 1996 and, in revised form, December 10, 1996 and January 31, 1997.

1991 Mathematics Subject Classification. Primary 11Q25.

(C) 1998 American Mathematical Society 
$\mathcal{M}(K)$ ), then for every nonconstant affine (resp. partial rational linear) function $h$, the couple $(h(S), h(T))$ also is a bi-URS for $\mathcal{A}(K)$ (resp. $\mathcal{M}(K))$.

In $\mathbb{C}$, Yi Hongxun proved the existence of URS's for $\mathcal{A}(K)$, with $n$ elements, for any $n \geq 15[15]$.

On the other hand, in [1] Adams and Strauss showed that for every couple $(a, b) \in K^{2}$, if $f, g \in \mathcal{A}(K)$ satisfy $f^{-1}(\{a\})=g^{-1}(\{a\})$ and $f^{-1}(\{b\})=g^{-1}(\{b\})$, then $f=g$. Actually, here, this shows that for every couple $(a, b),(\{a\},\{b\})$ is a bi-URS for entire functions.

Recently, in [17], Ping Li and Chung-Chun Yang showed that in $\mathbb{C}$ there exist bi-URS's for meromorphic functions of the form $(S,\{\infty\})$, where $S$ has 15 points, and they found URS's for meromorphic functions that only have 19 points. Next, Mues and Reinders obtained URS's for meromorphic functions of 13 points [16]. Finally, Frank and Reinders have obtained URS's for meromorphic functions of only 11 points [10].

Of course, a URS for entire functions must have at least 3 points, because given 2 points $a, b$, there does exist an affine function of the form $f(x)=c x+d$, with $c \neq 0$, such that $h(a)=b, h(b)=a$, and therefore, putting $S=\{a, b\}$, it is seen that for every entire function $f$, we have $E(f, S)=E(h \circ f, S)$.

In the same way, a URS for meromorphic functions must have at least 4 points, because given 3 points $a, b, c$, there does exists a partial rational linear function $h$ that permutes the set $S=\{a, b, c\}$ (in a nontrivial way) and therefore, for every meromorphic function $f$, we have $E(f, S)=E(h \circ f, S)$.

In [4], we characterized the URS's for polynomials, in any algebraically closed field $L$, showing that they are the finite sets which are unpermutable by any affine function other than the identity. Next, we proved that in $p$-adic analysis, there exist URS's of $n$ elements, for $\mathcal{A}(K)$, for any $n \geq 3$. Among sets of $n=3$ points, we proved that URS's for $\mathcal{A}(K)$ are just URS's for $K[x]$. This characterization of URS for $\mathcal{A}(K)$ has just been generalized for all $n \geq 3$ by W. Cherry and C.C. Yang [6].

Here, using certain lemmas proven in [4], and other specific properties of analytic elements [1], [9], [14], we will study bi-URS's for $\mathcal{M}(K)$, of the form $(S,\{w\})$.

Notation. In the following four theorems, $w$ denotes a point in $\widehat{K}$, and $h$ denotes the partial rational linear function defined as $h(x)=\frac{1}{x}+w$ if $w$ is in $K$, and $h=$ identity if $w=\infty$.

Theorem 1. Let $n, m \in \mathbb{N}$ be relatively prime and such that $n \geq m+2$ and $m \geq 5$. Let $a \in K^{*}$ satisfy $a^{n} \neq \frac{n^{n}}{m^{m}(n-m)^{n-m}}$. Then the polynomial $P(u)=$ $u^{n}-a u^{m}+1$ admits $n$ distinct zeros $z_{1}, \ldots, z_{n}$, and the set $S=h\left(\left\{z_{1}, \ldots, z_{n}\right\}\right)$ is such that $(S,\{w\})$ is a bi-URS for $\mathcal{M}(K)$.

Theorem 2. Let $n, m \in \mathbb{N}$ be relatively prime and satisfy $n \geq m+2$ and $m \geq 3$. Let $a \in K^{*}$ satisfy $a^{n} \neq \frac{n^{n}}{m^{m}(n-m)^{n-m}}$. Let $P(u)=u^{n}-a u^{m}+1$, and assume that for every $n-m$-th root $\zeta$ different from 1 , of $(-1)^{n-m}, P-\zeta$ has no zeros of order superior or equal to 2 . Then $P$ admits $n$ distinct zeros $z_{1}, \ldots, z_{n}$, and the set $S=h\left(\left\{z_{1}, \ldots, z_{n}\right\}\right)$ is such that $(S,\{w\})$ is a bi-URS for $\mathcal{M}(K)$. 
Theorem 3. Let $a \in K^{*}$ satisfy $a^{5} \neq \frac{3125}{108}$ and $a^{5} \neq \frac{3125}{27}$. Then the polynomial $P(u)=u^{5}-a u^{3}+1$ admits 5 distinct zeros $z_{1}, z_{2}, z_{3}, z_{4}, z_{5}$, and the set $S=$ $h\left(\left\{z_{1}, z_{2}, z_{3}, z_{4}, z_{5}\right\}\right)$ is such that $(S,\{w\})$ is a bi-URS for $\mathcal{M}(K)$.

Theorem 4. Let $a \in K^{*}$ satisfy $a^{6} \neq \frac{729}{16}$ and $a^{6} \neq \frac{729}{4}$. Then the polynomial $P(u)=u^{6}-a u^{4}+1$ admits 6 distinct zeros $z_{1}, z_{2}, z_{3}, z_{4}, z_{5}, z_{6}$, and the set $S=$ $h\left(\left\{z_{1}, z_{2}, z_{3}, z_{4}, z_{5}, z_{6}\right\}\right)$ is such that $(S,\{w\})$ is a bi-URS for $\mathcal{M}(K)$.

Corollary. For every $n \geq 5$ and for every $w \in \widehat{K}$, there exist bi-URS's for $\mathcal{M}(K)$ of the form $\left(\left\{z_{1}, \ldots, z_{n}\right\},\{w\}\right)$.

Remark 2. Taking into account the results obtained in [4] and [6], one may imagine that there exist URS's for $\mathcal{M}(K)$ with $n$ points for any $n \geq 4$, and a set of $n$ elements is a URS for $\mathcal{M}(K)$ if and only if it is unpermutable by any nonconstant partial rational linear function other than the identity. In the same way, one can think that there exist bi-URS's for $\mathcal{M}(K)$ of the form $\left(\left\{z_{1}, z_{2}, z_{3}\right\},\{\omega\}\right)$, and that a finite set $(S,\{\omega\})$ is a bi-URS for $\mathcal{M}(K)$ if and only if $S$ is unpermutable by any nonconstant partial rational linear function (different from the identity), admitting $\omega$ as a fixed point.

\section{THE PROOFS}

Let $a \in K$, and $r>0$. We will denote by $C(a, r)$ the circle $\{x \in K|| x-a \mid=r\}$, by $d(a, r)$ the disk $\{x \in K|| x-a \mid \leq r\}$, and by $d\left(a, r^{-}\right)$the disk $\{x \in K|| x-a \mid<r\}$. Inside a circle $C(a, r)$, we call a class of $C(a, r)$ any disk $d\left(b, r^{-}\right)$, with $b \in C(a, r)$. Given $s>r$, we will denote by $\Gamma(a, r, s)$ the annulus $\{x \in K|r<| x-a \mid<s\}$.

Notation. Let $L$ be an algebraically closed field, let $L[[X]]$ be the ring of power series with coefficients in $L$, and let $L((X))$ be its field of fractions.

Then every $f(X) \in K((X)) \backslash\{0\}$ is of the form $X^{q(f)} h(X)$, with $q(f) \in \mathbb{Z}$ and $h \in K[[X]]$, satisfying $h(0) \neq 0$. Then the mapping $\psi$ from $K((x))$ to $\mathbb{Z} \cup\{\infty\}$ defined as $\psi(f)=q(f)$ if $f \in K((x)) \backslash\{0\}$ and $\psi(0)=\infty$ is known to be a discrete ultrametric valuation.

Now, let $f \in \mathcal{M}(K)$. Since $\mathcal{M}(K)$ is clearly included in $K((X))$, the restriction of $\psi$ to $\mathcal{M}(K)$ defines a discrete ultrametric valuation. Besides, for each $\alpha \in K$, we may write $f \in \mathcal{M}(K)$ in the form $g(t)=f(\alpha+t)$, and consider the valuation $\omega_{\alpha}$ defined as $\omega_{\alpha}(f)=\psi(g)$.

Lemma 1 is quite elementary, and easily checked.

Lemma 1. Let $L$ be an algebraically closed field of characteristic 0 , let $a \in L^{*}$ and let $n, m \in \mathbb{N}$ satisfy $n>m>1$. Let $P(u)=u^{n}-a u^{m}+1 \in K[u]$, and let $\lambda \in L$. If $P-\lambda$ admits a zero $\theta$ of order $q \geq 2$, then $a$ and $\lambda$ must satisfy

(E) $\quad a^{n} \frac{(m)^{m}(n-m)^{n-m}}{n^{n}}=(1-\lambda)^{n-m}$.

Further, if $\lambda \neq 0$, if $P-\lambda$ admits a zero $\theta$ of order $q \geq 2$, and if $P-\frac{1}{\lambda}$ admits a zero $\theta^{\prime}$ of order $q^{\prime} \geq 2$, then $\lambda^{n-m}=(-1)^{n-m}$.

Definitions. A set $D$ is said to be infraconnected if for every $a \in D$, the mapping $I_{a}$ from $D$ to $\mathbb{R}_{+}$defined by $I_{a}(x)=|x-a|$ has an image whose closure in $\mathbb{R}_{+}$ is an interval. (In other words, a set $D$ is not infraconnected if and only if there exist $a$ and $b \in D$ and an annulus $\Gamma\left(a, r_{1}, r_{2}\right)$ with $0<r_{1}<r_{2}<|a-b|$ such that $\left.\Gamma\left(a, r_{1}, r_{2}\right) \cap D=\emptyset.\right)$ 
Given a closed bounded set $D$, the $K$-algebra of rational functions $h \in K(x)$ with no pole in $D$ is provided with the norm of uniform convergence on $D$, denoted by \|\|$_{D}$. The completion of $R(D)$ for this topology is a $K$-Banach algebra $H(D)$ whose elements are named the analytic elements in $D$ [7], [9], [14]. Lemma 2 is immediate.

Lemma 2. Let $D$ be a closed bounded set, and let $f \in \mathcal{M}(K)$ have no pole in $D$. Then $f$ belongs to $H(D)$.

We may extract the following lemma from classical results on continuous seminorms in $K$-Banach algebras $H(D)$ [8], [9], [11].

Lemma 3. Let $D$ be a closed bounded infraconnected set of diameter $s>0$, let $a \in D$, let $r \in] 0, s]$, and let $\mathcal{F}$ be the filter admitting for base the family of sets $D \cap$ $\left(\Gamma(a, l, r) \cup \Gamma\left(a, r, l^{\prime}\right)\right)$, with $\ell<r<\ell^{\prime}$. For every $f \in H(D),|f(x)|$ admits a limit, denoted by ${ }_{D} \varphi_{a, r}(f)$, along the filter $\mathcal{F}$, and the mapping ${ }_{D} \varphi_{a, r}$ is a multiplicative semi-norm on $H(D)$ satisfying ${ }_{D} \varphi_{a, r}(f) \leq\|f\|_{D}$. Furthermore, if $D$ contains the disk $d\left(a, r^{-}\right)$, then inside $d\left(a, r^{-}\right), f(x)$ is equal to a power series $\sum_{j=0}^{\infty} a_{j}(x-a)^{j}$, and satisfies ${ }_{D} \varphi_{a, r}(f)=\sup _{j \in \mathbb{N}}\left|a_{j}\right| r^{j}$.

Notation. By Lemma 3 the mapping $\phi_{r}$, defined in each $K$-algebra $H(d(0, r))$ as $\phi_{r}(f)={ }_{d(0, r)} \varphi_{0, r}$, is an absolute value on $H(d(0, r))$ which, in particular, applies to all $\mathcal{A}(K)$, and therefore has continuation $\bar{\phi}$ to the field $\mathcal{M}(K)$. For convenience, for all $f \in \mathcal{M}(K)$ we put $|f|(r)=\bar{\phi}_{r}(f)$.

So, by Lemma 3 we have Corollary a:

Corollary a. For every $r>0$ and every $f \in \mathcal{M}(K)$, we have

$$
|f|(r)=\lim _{|x| \rightarrow r,|x| \neq r}|f(x)| .
$$

Lemma 4. For any $f \in \mathcal{M}(K)$ and $r>0$, one has $\left|f^{\prime}\right|(r) \leq \frac{1}{r}|f|(r)$.

Proof. If $f$ belongs to $\mathcal{A}(K)$, this equality is classical ([9], Theorem 13.5). So, it is easily generalized to $\mathcal{M}(K)$. Indeed, let $h=\frac{f}{g} \in \mathcal{M}(K)$. Clearly, we have

$$
\left|h^{\prime}\right|(r) \leq \max \left(\frac{\left|f^{\prime}\right|(r)}{|g|(r)}, \frac{|f|(r)\left|g^{\prime}\right|(r)}{(|g|(r))^{2}}\right) \leq \max \left(\frac{|f|(r)}{r|g|(r)}, \frac{|f|(r)|g|(r)}{r(|g|(r))^{2}}\right)=\frac{|h|(r)}{r} .
$$

Lemma 5. Let $a \in K$, let $\Lambda=C(a, r)$ and let $D$ contain $\Lambda$. Any element $f \in$ $H(D)$ has finitely many zeros in $\Lambda$ and factorizes in the form $f=P g$ with $P$ the polynomial of the zeros of $f$ in $\Lambda$ and $g$ an element of $H(D)$ that has no zero in $\Lambda$. Besides $f$ satisfies $|f(x)| \leq{ }_{D} \varphi_{a, r}(f) \forall x \in C(a, r)$, and the equality $|f(x)|={ }_{D} \varphi_{a, r}(f)$ holds in all the classes of $C(a, r)$ that contain no zero of $f$.

Proof. Indeed, by Theorem 23.7 of [9], $f$ has finitely many zeros in $\Lambda$ and then this factorization is given by Theorem 14.5 of [9]. Besides by Theorem 23.6 of [9] we have $|h(x)|={ }_{D} \varphi_{a, r}(h) \forall x \in C(a, r),|P(x)| \leq{ }_{D} \varphi_{a, r}(P) \forall x \in C(a, r)$, and by Lemma 4.6 of [9] we have $|P(x)|={ }_{D} \varphi_{a, r}(P)$ for all $x$ in any class of $C(a, r)$ containing no zeros of $P$, so the conclusion is clear. 
Corollary b. Let $f \in \mathcal{M}(K)$, and let $\left(r c_{t}\right)_{t \in \mathbb{N}}$ be the sequence of radii of the circles containing at least one zero or one pole of $f$, with $r_{t}<r_{t+1} \forall t \in \mathbb{N}$. For all $t \in \mathbb{N}$, $\left(\alpha_{j}\right)_{1 \leq j \leq \nu_{t}}$ denotes the set of zeros of $f$ inside $C\left(0, r_{t}\right)$, and $\left(\beta_{j}\right)_{1 \leq j \leq \sigma_{t}}$ denotes the set of poles of $f$ inside $C\left(0, r_{t}\right)$. Let

$$
D=K \backslash\left(\bigcup_{t \in \mathbb{N}}\left(\bigcup_{j=1}^{\nu_{t}} d\left(\alpha_{j}, r_{j}^{-}\right)\right) \bigcup\left(\bigcup_{j=1}^{\sigma_{t}} d\left(\beta_{j}, r_{j}^{-}\right)\right)\right) .
$$

Then $D$ is infraconnected and we have $|f(x)|=|f|(|x|) \forall x \in D$.

Proof. Indeed, for every $r>0, f$ obviously belongs to $H(D \cap d(0, r))$.

Lemma 6. Let $f, g \in \mathcal{M}(K)$ and $s \in \mathbb{N}^{*}$ satisfy $\omega_{\alpha}(f) \geq s \omega_{\alpha}(g)$ for every $\alpha \in K$. Then there exists $h \in \mathcal{A}(K)$ such that $f=h g^{s}$.

Proof. Indeed, since each mapping $\omega_{\alpha}$ is a valuation on $\mathcal{M}(K)$, it is seen that $\frac{f}{g^{s}}$ has no pole, and therefore belongs to $\mathcal{A}(K)$.

Corollary c. Let $f, g \in \mathcal{M}(K)$ and $s \in \mathbb{N}^{*}$ satisfy $\omega_{\alpha}(f) \geq s \omega_{\alpha}(g)$ for every $\alpha \in K$. Then there exists $T>0$ such that $|f|(r) \geq T(|g|(r))^{s}$ for all $r \geq 1$.

Lemma 7. Let $D$ be a nonbounded infraconnected set, let $P(u) \in K[u]$ be a nonconstant monic polynomial and let $f \in \mathcal{M}(K)$ have no pole in $D$ and satisfy $\lim _{|x| \rightarrow \infty, x \in D} P(f(x))=0$. Then, there exists a zero $\theta$ of $P$ such that $\lim _{|x| \rightarrow \infty, x \in D} f(x)$ $=\theta$.

Proof. Let $P(u)=\prod_{j=1}^{t}\left(u-a_{j}\right)^{q_{j}}$, with $a_{j} \neq a_{l} \forall j \neq l$, and let $n=\operatorname{deg}(P)$. For every $\epsilon>0$, it is easily seen that if $|P(u)|<\epsilon^{n}$, then there exists an index $l \leq t$ such that $\left|u-\alpha_{l}\right|<\epsilon$. Now, let $\sigma=\min _{j \neq l}\left|a_{j}-a_{l}\right|$, and let $\left.\epsilon \in\right] 0, \sigma[$. There obviously exists $r>0$ such that $|P(f(x))|<\epsilon, \forall x \in D \backslash d(0, r)$. Let $E=D \backslash d(0, r)$, and for every $s>r$, let $E_{s}=E \cap d(0, s)$. Then, by our first remark, we have

$$
f(E) \subset \bigcup_{j=1}^{t} d\left(a_{j}, \epsilon\right) .
$$

We notice that $E$ is obviously infraconnected, and so is $E_{s}$, for every $s>r$. Therefore, since $f \in H\left(E_{s}\right), f\left(E_{s}\right)$ is also infraconnected (Theorem 21.12 of [9]) for every $s>r$. Thus, $f(E)$ is infraconnected as a union of an increasing family of infraconnected sets ([9], Corollary 2.7). Now, let $\alpha \in E$. By (1) there exists a zero $\theta$ of $P$ such that $|f(\alpha)-\theta| \leq \epsilon$.

We will show that $f(E) \subset d(\theta, \epsilon)$. Let $\beta \in E$, let $s=|\alpha-\beta|$, and suppose $|f(\alpha)-f(\beta)|>\epsilon$. There exists another zero $\zeta$ of $P$ such that $|f(\beta)-\zeta| \leq \epsilon$, hence we have $\zeta \neq \theta$, and therefore $|\theta-\zeta| \geq \sigma$, hence $|f(\beta)-f(\alpha)| \geq \sigma$. But then, by (1), we see that $\Gamma(\alpha, \epsilon, \sigma) \cap f(E)=\emptyset$. Hence this contradicts the fact that $f(E)$ is infraconnected and this ends the proof. 
Lemma 8. Let $S=\left\{z_{1}, \ldots, z_{n}\right\}$ be a set of order $n$ in $K$, and let $P(u)=\prod_{j=1}^{n}\left(u-z_{j}\right)$.

Let $f, g \in \mathcal{M}(K)$ have the same poles (taking multiplicities into account) and satisfy $E(f, S)=E(g, S)$. Then, there exists a constant $\lambda$ different from 0 , such that $P(f(x))=\lambda P(g(x))$ for all $x \in K$.

Proof. Let $\Sigma=E(f, S)$, and let $h(x)=\frac{P(f(x))}{P(g(x))}$. Let $\alpha$ be a zero of $P(f(x))$, and let $q$ be its order of multiplicity. Let $\theta=f(\alpha)$. Then $\theta$ obviously lies in $S$ and therefore $(\alpha, q)$ lies in $E(f, S)$. In the same way, this is also true for $g$, hence $(\alpha, q)$ lies in $E(g, S)$. So, both $P(f(x))$ and $P(g(x))$ admit each point $\alpha \in \Sigma$ as a zero, with the same order of multiplicity and they don't have any other zero. Hence, the only zeros (resp. poles) of $h$ are the poles of $P(g(x))$ (resp. of $P(f(x))$ ). But since $f, g$ have the same poles, taking multiplicities into account, it is seen that $P(g(x))$ and $P(f(x))$ have the same poles taking multiplicities into account. Finally, $h$ has neither any zero nor any pole, and therefore is a constant $\lambda$ obviously different from zero.

By Corollary 1 in [4], we have Proposition M:

Proposition M. Let $\alpha \in K$, and let $P \in K[u]$ satisfy:

i) $P(u)=c_{0}+\sum_{j=m}^{n} c_{j} u^{j}$, with $c_{0} c_{m} c_{n} \neq 0$, and $m>1$,

ii) $P^{\prime}$ has no multiple zero different from 0 .

Let $f, g \in \mathcal{M}(K)$ satisfy

iii) $P(f)=\lambda P(g)$ for some $\lambda \in K \backslash\{0,1\}$,

iv) $\omega_{\alpha}(g)>0$.

Then we have $\omega_{\alpha}(f)=0$, and $m \omega_{\alpha}(g)$ is equal either to $\omega_{\alpha}(f-f(\alpha))$, or to $2 \omega_{\alpha}(f-f(\alpha))$. Besides, if $m \omega_{\alpha}(g)=2 \omega_{\alpha}(f-f(\alpha))$, then we have $P^{\prime}(f(\alpha))=0$.

Notation. Let $\log$ be the real logarithm function of base $p>1$. Given $f \in \mathcal{M}(K)$, it will be convenient to use the valuation function $v(f, \mu)$, defined in $\mathbb{R}$, as $v(f, \mu)=$ $-\log \left(|f|\left(p^{-\mu}\right)\right)$. By classical results ([2], [9], [14]), it is well known that this function is continuous and piecewise linear.

Let $f \in \mathcal{M}(K)$ satisfy $f(0) \neq 0$ and $f(0) \neq \infty$, and let $f=\frac{g}{l}$, with $g, l \in \mathcal{A}(K)$, $g, l$ having no common zeros, and satisfying $l(0)=1$. In order to respect notations used in Nevanlinna's theory [3], for all $\mu \in \mathbb{R}$ we denote by $P(\mu, f)$ the number of poles of $f$ in $C\left(0, p^{-\mu}\right)$, taking multiplicities into account, and by $\bar{P}(\mu, f)$ the number of different poles of $f$ in $C\left(0, p^{-\mu}\right)$. Now, for all $\lambda \in \mathbb{R}$, we put

$$
\begin{aligned}
& M(\lambda, f)=\max (-v(f, \lambda), 0), \\
& N(\lambda, f)=\sum_{\mu \geq \lambda} P(\mu, f)(\mu-\lambda), \\
& \bar{N}(\lambda, f)=\sum_{\mu \geq \lambda} \bar{P}(\mu, f)(\mu-\lambda), \\
& T(\lambda, f)=M(\lambda, f)+N(\lambda, f) .
\end{aligned}
$$

Moreover, for all $a \in K$, we put $\Theta(a, f)=1-\limsup _{\lambda \rightarrow-\infty} \frac{\bar{N}\left(\lambda, \frac{1}{f-a}\right)}{T(\lambda, f)}$.

Lemma 9 is easily seen: 
Lemma 9. Let $f=\frac{g}{l} \in \mathcal{M}(K)$, with $l(0)=1$. Then

$$
T(\lambda, f)=-\min (v(l, \lambda), v(g, \lambda)) .
$$

Let $a \in K$ be different from 0 , and let $\bar{f}_{a}$ be the entire function whose zeros are of order one, and are all different zeros of $f-a$, satisfying $\bar{f}_{a}(0)=1$. Then we have $\bar{N}\left(\lambda, \frac{1}{f-a}\right)=-v\left(\bar{f}_{a}, \lambda\right)$.

By Theorem I.9 of [3], we have :

Proposition N. Let $f \in \mathcal{M}(K)$ satisfy $f(0) \neq 0$ and $f(0) \neq \infty$. The set $W$ of the $a \in K$ such that $\Theta(a, f) \neq 0$ is countable, and satisfies

$(\mathcal{N}) \quad \sum_{a \in W} \Theta(a, f) \leq 2$.

Now, thanks to Lemma 9, we will translate $(\mathcal{N})$ into terms of valuation. We put again $f=\frac{g}{l}$, with $g, l \in \mathcal{A}(K)$, and $l(0)=1$.

Let $a \in K$ be different from 0 , and let $\bar{f}_{a}$ be the entire function whose zeros are of order one, and are all the different zeros of $f-a$, satisfying $\bar{f}_{a}(0)=1$. Therefore we obtain:

(R) $\quad \Theta(a, f)=1-\limsup _{\lambda \rightarrow-\infty}\left(\frac{v\left(\bar{f}_{a}, \lambda\right)}{\min (v(l, \lambda), v(g, \lambda))}\right)$.

Lemma 10. Let $q \in \mathbb{N}^{*}$, let $\alpha \in K$ and let $f \in \mathcal{M}(K)$ be such that $f(0) \neq 0$ and $f(0) \neq \alpha$, and such that every zero of $f-\alpha$ has order superior or equal to $q$. Then, we have $\Theta(\alpha, f) \geq 1-\frac{1}{q}$.

Proof. Let $f=\frac{g}{l}$, with $g, l \in \mathcal{A}(K), g, l$ having no common zeros, and $l(0)=1$. Let $u=\bar{f}_{\alpha}$. Clearly $g-\alpha l$ is of the form $u^{q} h$, with $h \in \mathcal{A}(K)$. Hence we have $q v(u, \lambda)=v(g-\alpha l, \lambda)-v(h, \lambda)$. But of course,

$$
v(g-\alpha l, \lambda) \geq \min (v(g, \lambda), v(l, \lambda)+v(\alpha)),
$$

so we obtain: $v(u, \lambda) \geq \frac{1}{q} \min (v(g, \lambda), v(l, \lambda)+v(\alpha))-v(h, \lambda)$. By hypothesis, clearly, $f$ is not a constant. Hence we can find $\rho \in \mathbb{R}_{+}$such that $\min (v(g, \rho), v(l, \rho))$ $<0$, and then we have $\min (v(g, \lambda), v(l, \lambda))<0 \forall \lambda<\rho$. We put $\tau=-v(h, \rho)$, and take $\lambda>\rho$. Then, as $v(h, \lambda) \leq v(h, \rho)$ we obtain

$$
v(u, \lambda) \geq \frac{1}{q} \min (v(g, \lambda), v(l, \lambda)+v(\alpha))+\tau,
$$

and therefore

$$
\frac{v(u, \lambda)}{\min (v(g, \lambda), v(l, \lambda))} \leq \frac{\min (v(g, \lambda), v(l, \lambda)+v(\alpha))+\tau}{q \min (v(g, \lambda), v(l, \lambda))} .
$$

Clearly, neither $v(\alpha)$ nor $\tau$ have any incidence on the superior limit when $\lambda$ tends to $-\infty$. So, finally, we see that

$$
\limsup _{\lambda \rightarrow-\infty} \frac{v(u, \lambda)}{\min (v(g, \lambda), v(l, \lambda))} \leq \frac{1}{q},
$$

and then by $(\mathcal{R})$, the conclusion is clear. 
Notation. Given $q \in \mathbb{N}^{*}$, we denote by $G_{q}$ the group of $q$-th roots of 1 , and given $q, s \in \mathbb{N}$, we put $\mathcal{G}_{q, s}=\left(G_{q} \cup G_{s}\right) \backslash\left(G_{q} \cap G_{s}\right)$ (i.e.: the symmetric difference of $G_{q}$ and $\left.G_{s}\right)$.

Lemma 11. Let $a \in K^{*}$, and let $n, m \in \mathbb{N}$ with $n>m$. Let $f, g \in \mathcal{M}(K)$ be nonconstant and satisfy $f(x)^{n}-a f(x)^{m}=g(x)^{n}-a g(x)^{m}$ for all $x \in K$. Let $t$ be the cardinal of $\mathcal{G}_{n, m}$. If $t\left(1-\frac{1}{n-m}\right)>2$, then we have $f=g$.

Proof. Let $\zeta_{i}(0 \leq i \leq m-1)$ be the $m$-th roots of 1 , and let $\xi_{j}(0 \leq j \leq n-1)$ be the $n$-th roots of 1 . Suppose that $f$ is not equal to $g$. Let $h=\frac{f}{g}$. We can check that $g^{n-m}=\frac{a\left(h^{m}-1\right)}{h^{n}-1}$. Without loss of generality, by a change of origin, we may obviously assume that $h(0) \notin\left\{0, \zeta_{0}, \ldots, \zeta_{m-1}, \xi_{0}, \ldots, \xi_{n-1}\right\}$. If $h$ is a constant, one checks that so is $g^{n-m}$, and therefore so is $g$. Thus, without loss of generality, we may also assume that $h$ is not a constant. Then, we have

$$
g^{n-m}=\frac{a \prod_{i=0}^{m-1}\left(h-\zeta_{i}\right)}{\prod_{j=0}^{n-1}\left(h-\xi_{j}\right)} .
$$

Let $G_{m}^{\prime}=\mathcal{G}_{n, m} \cap G_{m}$, and let $G_{n}^{\prime}=\mathcal{G}_{n, m} \cap G_{n}$. Let $\zeta \in G_{m}^{\prime}$. Since $\zeta$ does not belong to $G_{n}$, each zero of $h-\zeta$ is a zero of $g^{n-m}$, and therefore is a zero of order at least $n-m$ of $h-\zeta$. In the same way, for each $\xi \in G_{n}^{\prime}$, as $\zeta$ does not belong to $G_{m}$, every zero of $h-\xi$ is a pole of $g^{n-m}$, and therefore is a zero of order at least $n-m$ of $h-\xi$. As a consequence, by Lemma 10 we have

$$
\Theta(\nu, h) \geq 1-\frac{1}{n-m} \quad \text { for every } \nu \in \mathcal{G}_{n, m} .
$$

Applying (2) to each element of $\mathcal{G}_{n, m}$, we obtain:

$$
\sum_{\nu \in \mathcal{G}_{n, m}} \Theta(\nu, h) \geq t\left(1-\frac{1}{n-m}\right),
$$

and then, by Proposition $\mathrm{N}$ we have $t\left(1-\frac{1}{n-m}\right) \leq 2$. This ends the proof.

Corollary d. Let $a \in K^{*}$, and let $n, m \in \mathbb{N}$ be relatively prime, with $n \geq m+2$ and $m \geq 3$. Let $f, g \in \mathcal{M}(K)$ be nonconstant and satisfy $f(x)^{n}-a f(x)^{m}=$ $g(x)^{n}-a g(x)^{m}$ for all $x \in K$. Then we have $f=g$.

Remark. In [5], we stated $(t-1)\left(1-\frac{1}{n-m}\right)>2$ instead of $t\left(1-\frac{1}{n-m}\right)>2$, considering that one of the values $\left\{\zeta_{0}, \ldots, \zeta_{m-1}, \xi_{0}, \ldots, \xi_{n-1}\right\}$ might be omitted by $h$. In fact, Proposition $\mathrm{N}$ does apply to all values, even to a value omitted by the function we consider. So, we don't have to do this restriction.

Lemma 12. Let $a \in K^{*}$ and let $n, m \in \mathbb{N}$ satisfy $n>m>2$. Let $\lambda \in K \backslash\{0,1\}$. Let $P(u)=u^{n}-a u^{m}+1 \in K[u]$. Let $f, g \in \mathcal{M}(K)$ satisfy $P(f(x))=\lambda P(g(x))$ and $P^{\prime}(f(\alpha)) \neq 0$ for each zero $\alpha$ of $g$. Then, there exists a constant $A>0$ such that $|f|(r) \geq A r(|g|(r))^{2} \forall r \geq 1$. 
Proof. First, we notice that $f$ and $g$ have the same poles, with the same order of multiplicity, respectively. Hence, we have $E(f, \infty)=E(g, \infty)$. By Proposition M, for every zero $\alpha$ of $g$, we have $\omega_{\alpha}\left(f^{\prime}\right)+1=m \omega_{\alpha}(g) \geq 3 \omega_{\alpha}(g)$, hence $\omega_{\alpha}\left(f^{\prime}\right) \geq$ $2 \omega_{\alpha}(g)$. Next, as $E(f, \infty)=E(g, \infty)$, it is seen that for every pole $\beta$ of $g$, we have $\omega_{\beta}\left(f^{\prime}\right) \geq 2 \omega_{\beta}(g)$. So, the inequality $\omega_{\alpha}\left(f^{\prime}\right) \geq 2 \omega_{\alpha}(g)$ holds for every $\alpha \in K$. As a consequence, by Corollary c there exists $A>0$ such that $\left|f^{\prime}\right|(r) \geq A|g|(r)^{2}$ for all $r \geq 1$, and therefore by Lemma 4 we have $|f|(r) \geq A r(|g|(r))^{2}$.

Proposition P. Let $a \in K^{*}$, let $n, m \in \mathbb{N}$ satisfy $n>m \geq 3$ and let $P(u)=$ $u^{n}-a u^{m}+1 \in K[u]$. Besides, when $m=3$ or $m=4$, we assume that for every $n-m$-th root $\zeta$ of $(-1)^{n-m}$ different from $1, P-\zeta$ only admits zeros of order 1 .

Let $\lambda \in K \backslash\{0,1\}$ and let $P(u)=u^{n}-a u^{m}+1 \in K[u]$. Let $f, g \in \mathcal{M}(K)$ satisfy

$$
P(f(x))=\lambda P(g(x)) \text {. }
$$

Then $\lambda=1$.

Proof. Let $\left(s_{q}\right)_{q \in \mathbb{N}}$ be the sequence of the radii of the circles containing at least one zero or one pole of $g$ or $f$ (with $s_{q}<s_{q+1}$ ). For each $q \in \mathbb{N}$, let $\left\{\alpha_{1}, \ldots, \alpha_{\nu_{q}}\right\}$ be the set of the zeros of $f$ and $g$ inside $C\left(0, s_{q}\right)$, and let $\left\{\beta_{1}, \ldots, \beta_{\sigma_{q}}\right\}$ be the set of the poles of $f$ and $g$ inside $C\left(0, s_{q}\right)$. We put

$$
D=K \backslash\left(\bigcup_{q \in \mathbb{N}}\left(\bigcup_{j=1}^{\nu_{q}} d\left(\alpha_{j}, s_{j}^{-}\right)\right) \bigcup\left(\bigcup_{j=1}^{\sigma_{q}} d\left(\beta_{j}, s_{j}^{-}\right)\right)\right) .
$$

Then $D$ is an infraconnected set, and therefore by Corollary b we have

$$
\begin{aligned}
& |f(x)|=|f|(|x|) \forall x \in D, \\
& |g(x)|=|g|(|x|) \forall x \in D .
\end{aligned}
$$

We suppose $\lambda \neq 1$. Then $f$ and $g$ have no common zero. In the same way, there exist no sequences $\left(y_{\ell}\right)_{\ell \in \mathbb{N}}$ in $D$ such that $\lim _{\ell \rightarrow \infty} f\left(y_{\ell}\right)=\lim _{\ell \rightarrow \infty} g\left(y_{\ell}\right)=0$. But by (4) we know that for every $r \in\left|K^{*}\right|$, in the circle $C(0, r)$ the equality $|f(x)|=|f|(r)$ holds in all the classes of $C(0, r)$, except in finitely many. As a consequence, in each circle $C(0, r)$, with $r \in\left|K^{*}\right|$ (since $C(0, r)$ admits infinitely many classes), there does exist $x \in C(0, r)$ such that $|f(x)|=|f|(r)$ and $|g(x)|=|g|(r)$. Thus, it is seen that we can't have $\lim _{r \rightarrow \infty}|f|(r)=\lim _{r \rightarrow \infty}|g|(r)=0$. Hence there exists a constant $M>0$ and an increasing sequence $\left(r_{\ell}\right)_{\ell \in \mathbb{N}}$ such that $r_{0} \geq 1$ and $\lim _{\ell \rightarrow \infty} r_{\ell}=+\infty$, satisfying at least one of the following two conditions:

$$
\begin{aligned}
& |f|\left(r_{\ell}\right) \geq \max \left(|g|\left(r_{\ell}\right), M\right) \forall \ell \in \mathbb{N}, \\
& |g|\left(r_{\ell}\right) \geq \max \left(|f|\left(r_{\ell}\right), M\right) \forall \ell \in \mathbb{N} .
\end{aligned}
$$

Henceforth, we will assume that (6) is satisfied.

First, we suppose $m \geq 5$. We will prove

$$
\omega_{\beta}\left(g^{\prime}\right) \geq 2 \omega_{\beta}(f) \quad \text { whenever } \beta \in K .
$$

By (3) we notice that $f$ and $g$ have the same poles, taking multiplicities into account. Hence, for every pole $\beta$ of $f$, we have $\omega_{\beta}\left(g^{\prime}\right)=\omega_{\beta}(f)-1 \geq 2 \omega_{\beta}(f)$, and of course, for every $\beta \in K$ such that $\omega_{\beta}(f)=0$ we have $\omega_{\beta}\left(g^{\prime}\right) \geq 2 \omega_{\beta}(f)=0$. 
Now, let $\alpha \in K$ be a zero of $f$. By Proposition M we have $2\left(\omega_{\alpha}\left(g^{\prime}\right)+1\right) \geq$ $m \omega_{\alpha}(f) \geq 5 \omega_{\alpha}(f)$. If $\omega_{\alpha}(f) \geq 2$, we see that $\frac{5}{2} \omega_{\alpha}(f)-1 \geq 2 \omega_{\alpha}(f)$, hence $\omega_{\alpha}\left(g^{\prime}\right) \geq$ $2 \omega_{\alpha}(f)$. And if $\omega_{\alpha}(g)=1$, we have $\omega_{\alpha}\left(g^{\prime}\right) \geq \frac{3}{2}$, hence $\omega_{\alpha}\left(g^{\prime}\right) \geq 2$, which finishes proving (8).

Then, by Corollary c, there exists a constant $B$ such that $\left|g^{\prime}\right|(r) \geq B(|f|(r))^{2} \forall r$ $\geq 1$. So, by Lemma 4 , we obtain

$$
|g|\left(r_{\ell}\right) \geq r_{\ell} B\left(|f|\left(r_{\ell}\right)\right)^{2}, \quad \text { whenever } \ell \in \mathbb{N} .
$$

But since $|f|\left(r_{\ell}\right) \geq M$ for every $\ell \in \mathbb{N}$, (9) shows that $\lim _{\ell \rightarrow \infty}|g|\left(r_{\ell}\right)=+\infty$. Hence by (6) we have

$$
\lim _{\ell \rightarrow \infty}|f|\left(r_{\ell}\right)=+\infty
$$

and therefore (9) shows that $|g|\left(r_{\ell}\right)>|f|\left(r_{\ell}\right)$ as soon as $r_{\ell} B\left(|f|\left(r_{\ell}\right)\right)^{2}>|f|\left(r_{\ell}\right)$. So, (6) is contradicted, and then this contradiction shows that $\lambda=1$.

Now, we suppose $3 \leq m \leq 4$. For every $t \in K$ we denote by $Q_{t}$ the polynomial $P(u)-t$. First, we suppose that neither $Q_{\lambda}$ nor $Q_{\frac{1}{\lambda}}$ admits any zero of order superior or equal to 2 . By Lemma 12 , there exists a constant $A \in] 0,+\infty[$ such that $|f|(r) \geq A r|g|(r)^{2}$ for every $r \geq 1$. But in the same way, considering the equality $P(g)=\frac{1}{\lambda} P(f)$, we have a constant $\left.B \in\right] 0,+\infty\left[\right.$ such that $|g|(r) \geq B r|f|(r)^{2}$ for every $r \geq 1$. So, we notice that relation (9) is satisfied again. Besides, we obtain $A B r^{2}|f g|(r) \leq 1 \forall r \geq 1$. In particular, we have $A B r_{\ell}^{2}|f g|\left(r_{\ell}\right) \leq 1 \forall \ell \in \mathbb{N}$. But by (6) and (9), it is seen that both $|f|\left(r_{\ell}\right)$ and $|g|\left(r_{\ell}\right)$ tend to $+\infty$ when $\ell$ tends to $+\infty$, and this contradicts $A B r^{2}|f g|\left(r_{\ell}\right) \leq 1$.

Now, we suppose that both $Q_{\lambda}$ and $Q_{\frac{1}{\lambda}}$ admit a zero of order superior or equal to 2 . Hence, by Lemma 1 we have $\lambda^{n-m^{\lambda}}=(-1)^{n-m}$, but this situation has been excluded by hypothesis when $3 \leq m \leq 4$.

Finally, we suppose that at least one of the two polynomials $Q_{\lambda}, Q_{\frac{1}{\lambda}}$ does not admit any zero of order superior or equal to 2 . Without loss of generality, we may obviously assume that $Q_{\lambda}$ does not admit any zero of order $t \geq 2$. Then, by Proposition M, for every zero $\alpha$ of $g$, we have $\omega_{\alpha}\left(f^{\prime}\right)+1=m \omega_{\alpha}(g) \geq 3 \omega_{\alpha}(g)$, hence $\omega_{\alpha}\left(f^{\prime}\right) \geq 2 \omega_{\alpha}(g)$. Next, as $E(f, \infty)=E(g, \infty)$, it is seen that for every pole $\beta$ of $g$, we have $\omega_{\beta}\left(f^{\prime}\right) \geq 2 \omega_{\beta}(g)$. So, the inequality $\omega_{\alpha}\left(f^{\prime}\right) \geq 2 \omega_{\alpha}(g)$ holds for every $\alpha \in K$. As a consequence, by Lemma 6 there exists $h \in \mathcal{A}(K)$ (h not identically zero), such that

$$
f^{\prime}=h g^{2}
$$

And there exists a constant $B>0$ such that

$$
\left|f^{\prime}\right|(r) \geq B|g|(r)^{2} \forall r \geq 1 \text {. }
$$

We will deduce

$$
\lim _{|x| \rightarrow+\infty, x \in D} g(x)=0 .
$$

Indeed, suppose that (13) is not true. So, we don't have $\lim _{r \rightarrow+\infty, x \in D}|g|(r)=0$, and therefore, by (4) there obviously exist $\delta>0$ and a sequence $\left(\rho_{\ell}\right)_{\ell \in \mathbb{N}}$ in $|K|$ such that $\rho_{0} \geq 1,|g|\left(\rho_{\ell}\right) \geq \delta$ for all $\ell \in \mathbb{N}$, and $\lim _{\ell \rightarrow+\infty} \rho_{\ell}=+\infty$. Now, by (4) and (5), we 
can find a sequence $\left(x_{\ell}\right)_{\ell \in \mathbb{N}}$ in $D$ such that $\left|x_{\ell}\right|=\rho_{\ell}$, and $\left|f\left(x_{\ell}\right)\right|=|f|\left(\rho_{\ell}\right)$ for all $\ell \in \mathbb{N}$. Hence, by Lemma 4 and by (12) we have

$$
\left|f\left(x_{\ell}\right)\right|=|f|\left(\rho_{\ell}\right) \geq \rho_{\ell}\left|f^{\prime}\right|\left(\rho_{\ell}\right) \geq B \rho_{\ell}\left(|g|\left(\rho_{\ell}\right)\right)^{2} \geq B \rho_{\ell}\left|g\left(x_{\ell}\right)\right|^{2} \quad \forall \ell \in \mathbb{N} .
$$

But since $|g|\left(\rho_{\ell}\right) \geq \delta \forall \ell \in \mathbb{N}$, clearly we have $\lim _{\ell \rightarrow+\infty}\left|f\left(x_{\ell}\right)\right|=+\infty$, and therefore by (14) we have $\lim _{\ell \rightarrow \infty} \frac{g\left(x_{\ell}\right)}{f\left(x_{\ell}\right)}=0$. As a consequence, it is seen that

$$
\lim _{\ell \rightarrow+\infty}\left(\frac{\lambda P\left(g\left(x_{\ell}\right)\right)-P\left(f\left(x_{\ell}\right)\right)}{f\left(x_{\ell}\right)^{n}}\right)=-1 .
$$

But this clearly contradicts the equality $P(f)=\lambda P(g)$, and finishes showing (13). Now, by deriving the basic relation, we have $f^{\prime}(x) P^{\prime}(f(x))=\lambda g^{\prime}(x) P^{\prime}(g(x))$, hence by (11) we obtain $h(x) g(x)^{2} P^{\prime}(f(x))=\lambda g^{\prime}(x) g(x)^{2}\left(n g(x)^{n-3}-a m g(x)^{m-3}\right)$, and finally

$$
h(x) P^{\prime}(f(x))=\lambda g^{\prime}(x)\left(n g(x)^{n-3}-a m g(x)^{m-3}\right) .
$$

We notice that for every $r>0$, as $D \cap d(0, r)$ only has finitely many holes, $g^{\prime}$ does belong to $H(D \cap d(0, r))$ ([9], Corollary 19.2). Let $g=\frac{g_{1}}{g_{2}}$, with $g_{1}, g_{2} \in \mathcal{A}(K)$, $g_{1}, g_{2}$ having no common zero. Clearly, by Lemma 5 , in $C(0, r) \cap D$ we have $\left|g_{2}(x)\right|=\left|g_{2}\right|(r)$, and of course, $\left|g_{1}^{\prime}(x) g_{2}(x)-g_{1}(x) g_{2}^{\prime}(x)\right| \leq\left|g_{1}^{\prime} g_{2}-g_{1} g_{2}^{\prime}\right|(r)$, so $\left|g^{\prime}(x)\right| \leq\left|g^{\prime}\right|(r)$. Then by Corollary b and Lemma 4, we obtain $\left|g^{\prime}(x)\right| \leq\left|g^{\prime}\right|(r) \leq$ $\frac{|g|(r)}{r} \leq|g|(r) \forall x \in C(0, r) \cap D$, for every $r \geq 1$. Then by (13), we have $\lim _{|x| \rightarrow+\infty, x \in D} g^{\prime}(x)=0$, hence by (15), we obtain $\lim _{|x| \rightarrow+\infty, x \in D} h(x) P^{\prime}(f(x))=0$. In particular, we have $\lim _{r \rightarrow+\infty}|h|(r)\left|P^{\prime}(f)\right|(r)=0$. But as $h \in \mathcal{A}(K)$, we obtain $\lim _{r \rightarrow+\infty}\left|P^{\prime}(f)\right|(r)=0$, hence $\lim _{|x| \rightarrow+\infty, x \in D} P^{\prime}(f(x))=0$. Now, by (13) we have $\lim _{|x| \rightarrow+\infty, x \in D} P(f(x))-\lambda=0$. As a consequence, by Lemma 7 there does exist a zero $\theta$ of $P-\lambda$ such that $\lim _{|x| \rightarrow+\infty, x \in D} f(x)=\theta$. Thus, we have $\lim _{|x| \rightarrow+\infty, x \in D} P(f(x))=$ $\lambda=P(\theta)$, while $\lim _{|x| \rightarrow+\infty, x \in D} P^{\prime}(f(x))=0$, hence $P^{\prime}(\theta)=0$, and therefore $\theta$ is a zero of $Q_{\lambda}$ of order $q \geq 2$, which just contradicts the hypothesis. This ends the proof.

Proofs of the theorems. We notice that the condition $a^{n} \frac{(m)^{m}(n-m)^{n-m}}{n^{n}} \neq 1$ is satisfied in each theorem. Hence, by Lemma $1, P$ has no zero of order greater than 1. First, we assume $w=\infty$. Then, by hypothesis, $f$ and $g$ have the same poles, taking multiplicities into account. So, by Lemma 8 , there exists a constant $\lambda$ different from 0 such that $P(f(x))=\lambda P(g(x))$ for all $x \in K$.

Now, we can check that Proposition P clearly applies to the hypotheses of both Theorem 1 and Theorem 2, and shows that $\lambda=1$. Next, suppose we are in the hypothesis of Theorem 3 or Theorem 4 . By Lemma 1, we can easily check that $P+1$ does not admit any zero of order $q \geq 2$, hence the hypotheses of Proposition $\mathrm{P}$ are satisfied again. Thus, in all cases, we have $\lambda=1$. Now we can show that $f=g$ thanks to Lemma 11 and Corollary d. Indeed, in Theorems 1,2,3 as $m, n$ are relatively prime, we check that $n \geq m+2$, and that $m \geq 3$, and then we may apply Corollary d. In Theorem 4 , as $K$ has characteristic zero, we check that the 
cardinal $t$ of $\mathcal{G}_{n, m}$ is 6 , hence we have $t\left(1-\frac{1}{n-m}\right) \geq \frac{5}{2}$, and then the conclusion is given by Lemma 11 .

Finally, we can easily generalize when $w \in K$. Indeed let $l=h^{-1}$, and let $S^{\prime}=l(S)$. So, we have $l(x)=\frac{1}{x-w}$ and $S^{\prime}$ is the set of zeros of $P$. Then we may apply to $\left(S^{\prime},\{\infty\}\right)$ our theorems already proven when $w=\infty$, and then $\left(S^{\prime},\{\infty\}\right)$ is a bi-URS for $\mathcal{M}(K)$. But, as $S=h\left(S^{\prime}\right)$, and $w=h(\infty)$, by Remark 1 (at the beginning of the article), $(S,\{w\})$ also is a bi-URS for $\mathcal{M}(K)$.

\section{REFERENCES}

1. Adams, W.W. and Straus, E.G. Non archimedian analytic functions taking the same values at the same points. Illinois J. Math. 15, 418-424 (1971). MR 43:3504

2. Amice, Y. Les nombres p-adiques. PUF (Paris, 1975). MR 56:5510

3. Boutabaa, A. Theorie de Nevanlinna p-adique. Manuscripta Mathematica 67, pp.251-269, (1990). MR 91m:30039

4. Boutabaa, A. Escassut, A. and Haddad, L. On uniqueness of p-adic entire functions. To appear in Indagationes Mathematicae (1997).

5. Boutabaa, A. and Escassut, A. Uniqueness of p-adic meromorphic functions. Comptes Rendus de l'Académie des Sciences, Paris, t; 325, Serie I, p. 571-575, 1997. CMP 98:02

6. W. Cherry and C.-C. Yang Uniqueness of non-Archimedean entire functions sharing sets of values counting multiplicities, to appear in the Proceedings of the AMS.

7. Escassut, A. Algèbres d'éléments analytiques en analyse non archimédienne, Indagationes Mathematicae, t.36, p. 339-351 (1974). MR 51:10671

8. Escassut, A. Elements analytiques et filtres percés sur un ensemble infraconnexe, Ann. Mat. Pura Appl. t.110 p. 335-352 (1976). MR 54:13132

9. Escassut, A. Analytic Elements in p-adic Analysis. World Scientific Publishing Co. Pte. Ltd. (Singapore, 1995). MR 97e:46106

10. Frank, G. and Reinders, M. A unique Range set for meromorphic functions with 11 eleven elements, to appear in Complex Variable.

11. Garandel, G. Les semi-normes multiplicatives sur les algèbres d'éléments analytiques au sens de Krasner, Indagationes Mathematicae 37, n4, p.327-341, (1975). MR 52:11112

12. Gross, F. Factorization of meromorphic functions and some open problems. Lecture Notes in pure and Applied Math. 78, 51-67 (1982).

13. Gross, F. -Yang C.C. On preimage and range sets of meromorphic functions. Proc. Japan Acad. 58 (1):17 (1982). MR 83d:30027

14. Krasner, M. Prolongement analytique uniforme et multiforme dans les corps valués complets. Les tendances géométriques en algèbre et théorie des nombres, Clermont-Ferrand, p.94141 (1964). Centre National de la Recherche Scientifique (1966), (Colloques internationaux du C.N.R.S. Paris, 143). MR 34:4246

15. Yi, H. On a question of Gross. Science in China Vol. 38 No. 1 (1995). MR 96h:30054

16. Mues, E. and Reinders, M. Meromorphic functions sharing one value and unique range sets. Kodai Math. J. 18, p. 515-522, (1995). MR 97f:30044

17. Li, P. and Yang, C.C. On the unique range set of meromorphic functions. Proceedings of the AMS, Volume 124, Number 1, pp. 177-185 (1996). MR 96d:30033

Laboratoire de Mathématiques Pures, Université Blaise Pascal, (Clermont-Ferrand), Les Cézeaux, 63177 Aubiere Cedex, France

E-mail address: boutabaa@ucfma.univ-bpclermont.fr

E-mail address: escassut@ucfma.univ-bpclermont.fr 